ce au mésenchyme hépatique et qu'il joue un rôle majeur dans l'induction de la différenciation hépatocytaire. On peut donc désormais étendre ces observations et proposer que les cellules endothéliales provenant du mésoderme cardiaque jouent un tel rôle. De plus, les cellules endothéliales jouent un rôle inducteur beaucoup plus général dans de nombreux systèmes d'origine endodermique. On peut aussi se demander si un tel rôle trophique des cellules endothéliales ne concerne pas des organes provenant d'autres feuillets embryonnaires. Les données cellulaires présentées cidessus sont importantes et on peut pen- ser que, dans un avenir assez proche, les études porteront sur la caractérisation des différents signaux et sur l'identification des molécules impliquées dans l'induction, par les cellules endothéliales, de la différenciation de l'endoderme. $\diamond$ Endoderm différentiation under the control of endothelial cells?

\title{
NOUVELL
}

\section{La kinase FRAP/mTOR : une nouvelle cible dans le traitement des cancers dépendants de la voie PI3-kinase/PTEN

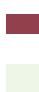

Catherine Greenland, Georges Delsol, Bernard Payrastre
> Une série d'articles récents [1-4] suggère qu'une cellule cancéreuse peut devenir très dépendante d'une voie de signalisation qui s'est hiérarchiquement imposée au cours des processus d'installation et de progression tumorales. L'exemple illustré dans ces travaux concerne des cellules tumorales présentant une sur-activation de la voie phospho-inositide 3 -kinase (PI3-kinase) conduisant à la stimulation de mTOR, une protéine kinase impliquée dans le contrôle de la traduction des ARN messagers. Ces cellules deviennent extrêmement sensibles aux inhibiteurs de mTOR (rapamycine ou $\mathrm{CCl}$-779) qui, utilisés à faible dose, conduisent à une réduction spécifique de la taille des tumeurs en bloquant la prolifération et en affectant la taille des cellules cancéreuses.

\section{PI3-kinases et cancer}

Les PI3-kinases sont une famille d'enzymes qui synthétisent des seconds messagers lipidiques, les D3-phosphoinositides. Ces phospho-inositides jouent un rôle majeur dans la régulation de plu- sieurs réponses cellulaires, notamment la prolifération et la survie [5-7]. En se liant spécifiquement à des domaines protéiques fonctionnels (domaines $\mathrm{PH}$, pleckstrin homology, FyVE ou PhoX), les D3-phospho-inositides permettent l'ancrage membranaire de protéines cytosoliques, organisant ainsi de manière spatio-temporelle l'activation de grandes voies de signalisation intracellulaire $[5,7]$. Le taux de ces seconds messagers lipidiques est strictement contrôlé par I'équilibre entre des PI 3-kinases qui les synthétisent et des phosphatases qui les hydrolysent de façon spécifique [7]. Or, plusieurs enzymes du métabolisme des D3-phospho-inositides, notamment les PI3-kinases de classe IA, sont impliquées dans des pathologies humaines [6], et notamment le développement de cancers $[5,6]$. Elles interviennent en tant qu'intermédiaires dans la transmission de signaux mitogéniques et de survie, mais sont aussi indispensables à l'effet transformant de plusieurs oncogènes à activité tyrosine kinase $[5,6]$. Certaines tumeurs humaines sont associées à une amplification du gène codant pour une $\mathrm{PI}$ 3-kinase de classe IA, ou à une surexpression et une activation de cette lipide kinase $[5,6]$. Aucune mutation activatrice de PI3-kinase n'a encore été rapportée dans des cancers humains. Toutefois, l'oncogène rétroviral $v-p 3 k$, un orthologue actif de la PI3-kinase de classe IA, a été isolé du sarcoma virus aviaire ASV16 qui induit des hémangiosarcomes chez le poulet $[5,6]$. Plusieurs enzymes stimulées en aval de la PI3-kinase, notamment le proto-oncogène Akt, sont également surexprimées et/ou activées dans divers types de cancers humains $[5,6]$. Les PI 3 -kinases de classe IA ou des protéines activées en aval pourraient donc être des cibles dans le traitement d'un certain nombre de cancers [6].

Une alternative pour activer les voies dépendantes des PI3-kinases est d'empêcher la dégradation des seconds messagers lipidiques produits par cette enzyme, notamment le phosphatidylinositol 3,4,5trisphosphate (Ptdlns $[3,4,5] \mathrm{P}_{3}$ ) et le phosphatidylinositol 3,4-bisphosphate (Ptdlns $\left.[3,4] P_{2}\right)$. Le gène $P T E N$, considéré comme un suppresseur de tumeur, code pour une enzyme à activité protéine tyrosine phosphatase et lipide phosphatase. PTEN est en quelque sorte une « anti-PI3kinase » puisqu'elle est capable d'hydrolyser le phosphate en position $3 \mathrm{du}$ Ptdlns $(3,4,5) P_{3}$ et du Ptdlns $(3,4) P_{2}$, une 
fonction indispensable à son activité de suppresseur de tumeur [8]. Or, des mutations germinales de PTEN, associées au développement de maladies telles que la maladie de Cowden, augmentent fortement le risque de développement de tumeurs, en particulier du sein et de la thyroïde [8-10]. De plus, des mutations somatiques de PTEN sont fréquemment associées à des cancers humains tels que les glioblastomes et les adénocarcinomes de la prostate, de l'endomètre et de l'ovaire [9]. A l'inverse, la surexpression de PTEN peut conduire à un arrêt de la prolifération cellulaire et à l'apoptose [10].

D'un point de vue moléculaire, un défaut d'activité de PTEN conduit à une accumulation intracellulaire de Ptdlns $(3,4,5) P_{3}$. Ce lipide va alors contribuer à l'activation de plusieurs protéines contenant des domaines $\mathrm{PH}$, notamment les kinases PDK et Akt $[5,7]$. L'expression d'une forme active de la sérine/thréonine kinase Akt peut prévenir plusieurs des effets de PTEN, suggérant que cette kinase est l'un des effecteurs principaux de la voie PI3-kinase/PTEN dans le contrôle de la prolifération et de la survie cellulaire [10]. Cependant, bien qu'Akt apparaisse comme une cible pharmacologique de choix, il n'existe à ce jour aucun inhibiteur spécifique de cette kinase.

mTOR, une cible pharmacologique dans le traitement des tumeurs présentant un dysfonctionnement de la voie PI3kinase /PTEN

En utilisant des souris déficientes en PTEN, deux équipes indépendantes ont cherché à optimiser le traitement des cancers liés à un dysfonctionnement de la voie PI 3-kinase /PTEN en ciblant des élé-

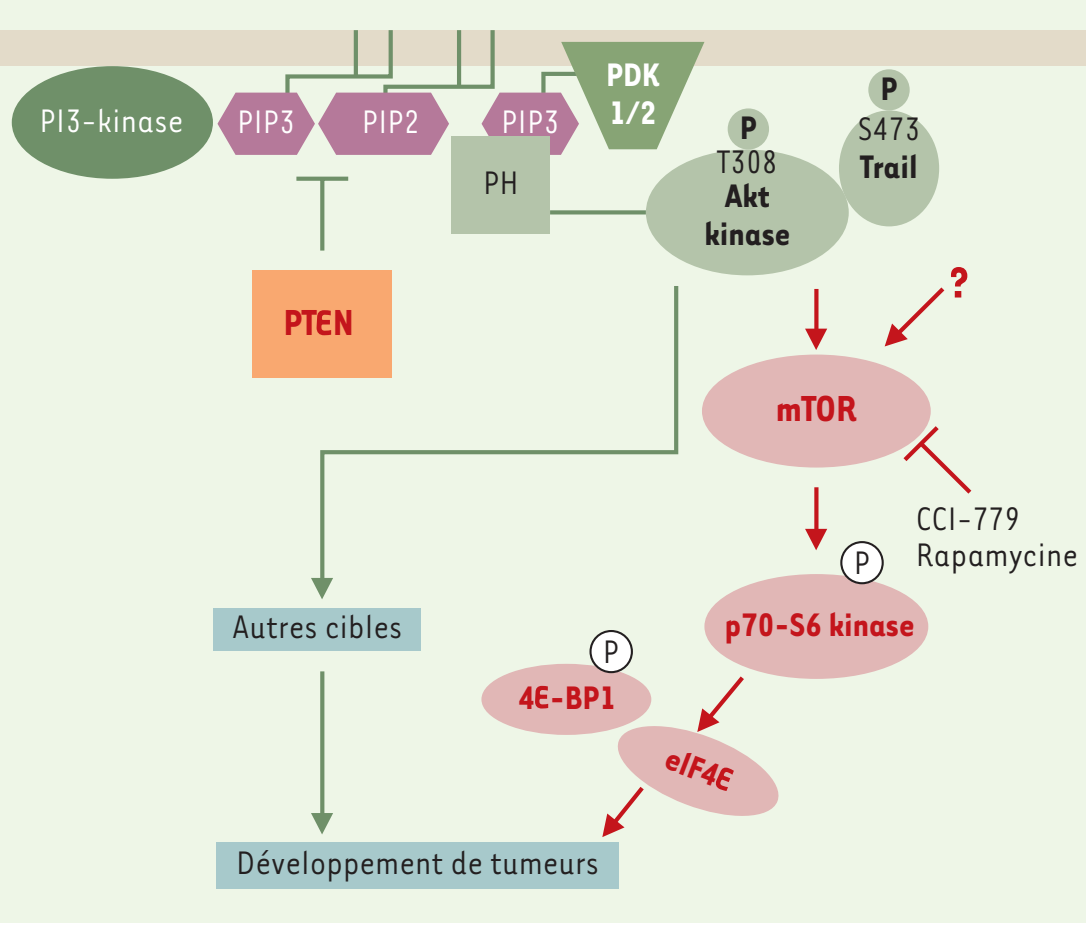

Figure 1. mTOR, une nouvelle cible dans le traitement des tumeurs présentant un dysfonctionnement de la voie PI 3-kinase/PTEN. En réglant le taux de Ptdlns $(3,4,5) \mathrm{P}_{3}$ (PIP3) et Ptdlns $(3,4)$ $P_{2}$ (PIP2), PTEN joue un rôle critique dans le contrôle des voies de signalisation dont l'activation dépend de ces seconds messagers lipidiques, notamment l'activation d'Akt. L'inhibition de mTOR, une kinase impliquée dans la voie PI 3-kinase/Akt, conduit au blocage préférentiel des cellules tumorales déficientes en PTEN. ments clés de cette cascade de signalisation $[1,2]$. Ils ont d'abord observé que l'absence de PTEN était associée à une activation d'Akt ainsi qu'à une surexpression, phosphorylation et activation des kinases mTOR et p70-S6. Ces cellules présentent également une phosphorylation de $4 \varepsilon-B P l$ corrélée à une prolifération accrue. En l'absence d'inhibiteur d'Akt, les auteurs ont utilisé un inhibiteur de mTOR, le CCl-779, un analogue de la rapamycine (un antibiotique aux propriétés immunosuppressives). À de faibles doses, cet agent diminue significativement la phosphorylation de p70-S6 kinase et de 4E-BPl in vitro et in vivo sans affecter l'activité d'Akt. Cet effet est associé à un arrêt de la progression tumorale. Les auteurs suggèrent que les cellules déficientes en PTEN deviennent dépendantes de la voie PI 3-kinase pour proliférer, ce qui augmente leur sensibilité au CCl-779 qui peut alors être utilisé efficacement à de faibles doses sans toxicité apparente pour les cellules normales. L'arrêt du traitement conduit à une reprise du processus tumoral indiquant un effet cytostatique du CCl- 779 .

Ces résultats placent la kinase mTOR et l'une de ses cibles, la p70-S6 kinase, dans la voie de signalisation conduisant au développement de tumeurs chez les souris déficientes en PTEN (Figure 1). D'autres travaux récents suggèrent que l'adressage d'Akt à la membrane plasmique peut conduire à l'activation de la p70-S6 kinase et à la phosphorylation de $4 \varepsilon-B P l$ entraînant une augmentation de la synthèse protéique. En effet, la phosphorylation de $4 \varepsilon-B P 1$ entraîne la libération du facteur d'initiation de la traduction des ARN messagers, elF4E, qui pourra alors pleinement fonctionner [5]. Cependant, les mécanismes moléculaires qui sous-tendent l'effet d'Akt sur p70-S6 kinase et la phosphorylation de $4 \varepsilon-B P 1$ restent mal connus. Bien que la kinase mTOR puisse être activée indépendamment de la voie $\mathrm{PI} 3$-kinase, il est vraisemblable qu'Akt puisse phosphoryler et activer directement mTOR qui, à son tour, 
contribuera à l'activation de la p70-S6 kinase.

mTOR apparaît donc comme une cible pharmacologique de première importance dans le traitement de tumeurs dans lesquelles l'équilibre PI 3 kinase/PTEN est modifié en faveur de la PI 3-kinase. Un diagnostic moléculaire fondé sur l'analyse des mutations de PTEN ainsi que sur le niveau de phosphorylation de protéines impliquées dans la voie PI 3-kinase/PTEN (p70-S6 kinase ou $4 \varepsilon-B P 1$ par exemple) pourrait permettre d'identifier les patients susceptibles de répondre au $\mathrm{CCl}-779$ (actuellement en phase I d'essai clinique dans divers cancers). L'association du CCl-779 et d'un agent cytotoxique pourrait représenter une nouvelle approche dans le traitement de certains cancers $[3,4]$.

Au-delà de cet exemple, il apparaît aujourd'hui important de rechercher la dépendance potentielle d'une cellule cancéreuse pour une voie de

signalisation particulière afin de concevoir des inhibiteurs pharmacologiques les plus ciblés et les moins toxiques possibles. $\diamond$ The FRAP/mTOR kinase: a novel target for anticancer drugs?

1. Neshat MS, Mellinghoff IK, Tran C, et al. Enhanced sensitivity of PTENdeficient tumors to inhibition of FRAP/mTOR. Proc Natl Acad Sci USA 2001 ; 98 : 10314-9.

2. Podsypanina K, Lee RT, Politis C, et al. An inhibitor of mTOR reduces neoplasia and normalizes p70/S6 kinase activity in Pten ${ }^{+}$mice. Proc Natl Acad Sci USA 2001 ; 98 : 10320-5.

3. Mills GB, Lu Y, Kohn EC. Linking molecular therapeutics to molecular diagnostics : inhibition of the FRAP/RAFT/TOR component of the PI3K pathway preferentially blocks PTEN mutant cells in vitro and in vivo. Proc Natl Acad Sci USA 2001 ; 98 : 10031-3.

4. Brooksbank C. Therapeutic targets: withdrawal symptoms. Nat Rev Cancer 2001 ; 1 : 6-11.

5. Vanhaesebroeck $B$, Leevers S), Khatereh A, et al. Synthesis and function of 3-phosphorylated inositol lipids. Annu Rev Biochem 2001 ; 70 : 535-602.

6. Stein RC, Waterfield MD. PI 3-kinase inhibition : a target for drug development? Mol Med Today 2000 ; 6 : 347-57.

7. Payrastre B, Missy K, Giuriato $S$, et al. Phosphoinositides : key players in cell signalling, in time and space. Cell Signal $2001 ; 13: 377-87$.

8. Di Cristofano A, Pandolfi PP. The multiples roles of PTEN in tumor suppression. Cell 2000 ; 100 : 387-90.

9. Ali IU, Schriml LM, Dean M. Mutational spectra of PTEN/MMAC gene : a tumor suppressor with lipid phosphatase activity. J Natl Cancer Inst 1999 ; 91 : 1922-32.

10. Maehama T, Taylor GS, Dixon JE. PTEN and myotubularin : novel phosphoinositide phosphatases. Annu Rev Biochem 2001 ; 70 : 247-79

\section{NOUVELLE}

\section{Le collagène de type XVIII, un nouveau collagène impliqué dans I'arborisation épithéliale rénale et pulmonaire}

Brigitte Lelongt, Pierre Ronco

> La première étape du développement rénal est caractérisée par l'invasion d'un amas de cellules mésenchymateuses indifférenciées par une structure épithéliale, le bourgeon urétéral. Des interactions séquentielles et réciproques entre ces deux tissus sont indispensables au déroulement harmonieux des étapes ultérieures du développement. Le bourgeon urétéral se divise d'abord en forme de T, puis ses branches s'allongent et se divisent de façon dichotomique pour engendrer l'ensemble du système collec- teur (morphogenèse ramifiée). Le mésenchyme va être converti en épithélium et se différencier pour former les composants épithéliaux du néphron à l'exception des canaux collecteurs (Figure 1) [1].

La matrice extracellulaire est impliquée dans la morphogenèse rénale à trois titres. Elle constitue un substrat physique permettant l'organisation spatiale des cellules, contrôlant ainsi la morphogenèse ramifiée du bourgeon urétéral. Elle joue également un rôle plus
Inserm U489, Hôpital Tenon, 4 , rue de la Chine, 75970 Paris Cedex 20, France.

actif par sa fonction de réservoir des facteurs de croissance libérés par des protéases [2]. Elle interagit enfin avec des récepteurs membranaires, les intégrines, activant ainsi des voies de signalisation intracellulaire.

Le collagène de type XVIII est un collagène récemment identifié dans les membranes basales [3]. Sa structure laissait supposer qu'il pouvait être impliqué dans la morphogenèse puisqu'il possède un 